\title{
Endothelial smoothened-dependent hedgehog signaling is not required for sonic hedgehog induced angiogenesis or ischemic tissue repair
}

\author{
Rajesh Gupta ${ }^{1,2} \cdot$ Alexander R. Mackie $^{2} \cdot$ Sol Misener ${ }^{2} \cdot$ Lijun Liu $^{1} \cdot$ Douglas W. Losordo $^{2,3} \cdot$ Raj Kishore ${ }^{2,4}$
}

Received: 6 September 2017 / Revised: 6 December 2017 / Accepted: 22 December 2017 / Published online: 16 February 2018

(c) United States \& Canadian Academy of Pathology 2018

\begin{abstract}
Sonic Hedgehog (Shh) signaling induces neovascularization and angiogenesis. It is not known whether the hedgehog signaling pathway in endothelial cells is essential to angiogenesis. Smoothened (Smo) transduces hedgehog signaling across the cell membrane. This study assessed whether endothelial Smoothened-dependent Shh signaling is required for Shhmediated angiogenesis and ischemic tissue repair. Endothelial-specific smoothened knockout mice, eSmo ${ }^{\text {Null }}$ were created using Cre-lox recombination system. eSmo ${ }^{\text {Null }}$ mice had no observable phenotype at baseline and showed normal cardiac function. Smoothened in CD31+ cells isolated from $\mathrm{eSmo}^{\text {Null }}$ hearts was significantly reduced compared to CD31+ cells from eSmo ${ }^{\mathrm{WT}}$ littermate control hearts. Fluorescence immunostaining of eSmo ${ }^{\text {Null }}$ heart sections showed Smo expression in endothelial cells was abolished. The hind-limb ischemia (HLI) model was used to assess the response to ischemic injury. Perfusion ratio, limb motor function, and limb necrosis were not significantly different after HLI between eSmo ${ }^{\text {Null }}$ mice and $\mathrm{eSmo}^{\mathrm{WT}}$. Capillary densities in the ischemic limb in $\mathrm{eSmo}^{\text {Null }}$ mice were also similar to eSmo ${ }^{\mathrm{WT}}$ at 4 weeks after HLI. Next, response to exogenous Shh was assessed in the corneal angiogenesis model. There was no significant difference in corneal angiogenesis induced by administration of Shh pellets between eSmo ${ }^{\mathrm{WT}}$ and $\mathrm{eSmo}^{\text {Null }}$ mice. Furthermore, in vitro experiments demonstrated that direct Shh had limited effects on endothelial cell proliferation and migration. However, conditioned media from Shh-treated fibroblasts had a more potent effect on endothelial cell proliferation and migration than non-treated conditioned media. Furthermore, Shh treatment of fibroblasts dramatically stimulated angiogenic growth factor expression, including PDGF-B, VEGF-A, HGF and IGF. PDGF-B was the most upregulated and may contribute to the large neo-vessels associated with Shh-induced angiogenesis. Taken together, these data demonstrate that Shh signaling via Smoothened in endothelial cells is not required for angiogenesis and ischemic tissue repair. Shh signaling via stromal cells likely mediates its angiogenic effects.
\end{abstract}

Rajesh Gupta

rajesh.gupta@utoledo.edu

1 Department of Medicine, College of Medicine and Life Sciences, University of Toledo, Toledo, OH, USA

2 Feinberg Cardiovascular Research Institute, Northwestern University, Chicago IL USA

3 Caladrius Biosciences Inc., Basking Ridge, NJ, USA

4 Center for Translational Medicine and Department of Pharmacology, Temple University School of Medicine, Philadelphia, PA, USA

\section{Introduction}

The Hedgehog (Hh) family of secreted proteins play an important role in cell growth, survival, and fate [1]. They are morphogens in multiple tissues during embryonic development [2]. Sonic Hedgehog (Shh) is the most widely expressed Hedgehog protein. It has a critical role in the development of the neural and vascular systems [3, 4]. In addition, Shh mediates key processes in neovascularization in response to injury, angiogenesis, and carcinogenesis in adults [5-8] Initial reports of the angiogenic effect of sonic hedgehog implicated an indirect mechanism where Shh stimulated the secretion of angiogenic growth factors [6]. More recent work has implicated a direct effect on the endothelium via a non-canonical signaling pathway involving Rho Kinase [9, 10]. 
The Shh receptor system consists of two major cell surface proteins, Patched-1 (Ptc-1) and Smoothened (Smo) [4]. Ptc-1 is the receptor for Shh, whereas Smo is the signal transducer. Ptc-1 tonically inhibits Smo, but once Shh binds to Ptc-1, Smo is disinhibited and downstream signaling occurs, classically through the activation of the gliomaassociated oncogene homolog (Gli) family of transcription factors [11].

Smo is a Frizzled-class 7-transmembrane protein composed of a heptahelical transmembrane domain (TMD) and an extracellular cysteine-rich domain (CRD) [12]. It has been shown that Shh signaling improves recovery after ischemic injury in mouse models of hind limb ischemia (HLI) and myocardial infarction [7, 13]. In vitro findings showed direct angiogenic effect of Shh via Rho kinasedependent signaling on endothelial cells [9]. However, the in vivo significance of endothelial Shh signaling and the mechanism by which Shh exerts an angiogenic effect have remained unclear $[6,9,10]$.

On the basis of robust evidence for the role of Shh in angiogenesis and vasculogenesis, and the in vitro findings of direct angiogenic effect on endothelial cells, we hypothesized that direct hedgehog signaling via endothelial Smo would be necessary for Shh-mediated angiogenesis and ischemic tissue repair. Therefore, we generated genetic, tissue-specific mouse knockout models utilizing Cre-lox technology to explore the in vivo role of direct endothelial hedgehog signaling via Smo in Shh-mediated angiogenesis and ischemic tissue repair. Surprisingly, we found that endothelial hedgehog signaling via Smo is dispensable for Shh-mediated angiogenesis and ischemic tissue repair. Furthermore, in vitro studies implicate a critical role of the stromal compartment in mediating the angiogenic response to Shh.

\section{Materials and methods}

\section{Animals}

Tie2-Cre mice (stock\# 008863) and mice with a floxed allele of Smoothened (stock\# 004526) obtained from Jackson Laboratory (Bar Harbor, ME), were bred to generate

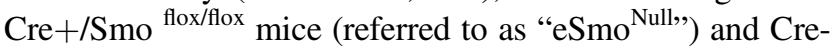
Smo flox/flox (referred to as "eSmowT") [14, 15]. Mice were housed in a sterile barrier facility. This study was carried out in strict accordance with the recommendations in the Guide for the Care and Use of Laboratory Animals of the National Institutes of Health. The protocol was approved by the Committee on the Ethics of Animal Experiments of Northwestern University. All surgery was performed under anesthesia, and all efforts were made to minimize suffering. Littermates were used for controls in all experiments. Both groups of mice were born with expected Mendelian frequencies and appeared normal. Routine PCR genotyping was performed on tail DNA. The following primers were used: Smo: forward, 5'-CTTGGGTGGAGAGGCTATTC$3^{\prime}$ and reverse, 5'-AGGTGAGATGACAGGAGATC-3'; Cre: forward, 5'-TGCCTGCATTACCGGTCGATGC-3' and reverse, 5'-CCATGAGTGAACGAACCTGGTCG-3'.

\section{Mouse hind-limb ischemia model}

Hind-Limb Ischemic (HLI) was performed in 8- to10-weekold male mice as described previously [16]. Briefly, the left femoral artery was exposed from the proximal end of the femoral artery to the distal portion of the saphenous vein, then the femoral artery and all side-branches were dissected and excised, and the overlying skin was closed with sutures. Critical limb ischemia was verified postoperatively by Laser Doppler Perfusion Imaging (LDPI, Moor LDI-Sim, Moor Instruments) to ensure the LDPI ratio (ischemic / nonischemic limb) was $\leq 0.20$. For follow-up, animals underwent reevaluations with LDPI at postoperative day 3, 7, 14, 21 , and 28. At each time point, tissue perfusion was measured via LDPI and reported as the ratio of ischemic / nonischemic limb. All LDPI measurements were taken on a $37^{\circ} \mathrm{C}$ heating pad to control body temperature.

For limb functional assays, limb motor function was scored as follows: (1) no limb use; (2) no foot use, limb use only; (3) restricted foot use; (4) no active toe use (spreading), foot use only; and (5) unrestricted limb use. Tissue salvage score was scored as follows: (1) limb amputation; (2) foot amputation; (3) total toe amputation; 4, partial toe amputation; 5, minor necrosis, nail loss only; (6) full recovery.

On the day of sacrifice, mice were injected with $50 \mu \mathrm{L}$ fluorescien-BS1-Lectin I (Vector Laboratories Inc.) via tail vein and sacrificed at least $15 \mathrm{~min}$ later. Tibialis anterior muscles were collected, fixed in methanol, paraffinembedded, and cut into 5- $\mu \mathrm{m}$ sections. Capillary density was evaluated in sections stained for the expression of BSlectin, an EC-specific marker. Perfused capillaries were identified by positive staining for BS-lectin. Sections were viewed at $\times 40$ magnification and vessels were counted in 10 high-power fields per section.

For HLI specimens, skeletal muscle immunohistochemistry was performed to identify capillaries. Mice were identified by injecting mice with BS-1 lectin (Vector Laboratories) $10 \mathrm{~min}$ before killing, and then sections were stained with anti-lectin primary antibodies (Vector Laboratories) and biotinylated anti-goat IgG (Vector Laboratories).

\section{Mouse corneal angiogenesis assay}

Shh Pellets were prepared as previously described [17]. Briefly, Shh pellets were prepared by mixing $10 \mu \mathrm{g}$ of Shh 
protein (Curis Inc., Cambridge, MA, USA) diluted in $10 \mu \mathrm{L}$ sterile PBS with $5 \mathrm{mg}$ sucrose octasulfate-aluminum complex (Sigma-Aldrich Co.). $10 \mu \mathrm{L}$ of $12 \%$ hydron in ethanol was added, and the suspension was deposited on a $400 \mu \mathrm{M}$ nylon mesh (Sefar America Inc., Depew NY, USA), then both sides of the mesh were covered with a thin layer of hydron and allowed to dry. eSmo ${ }^{\text {Null }}$ and $\mathrm{eSmo}^{\mathrm{WT}}$ mice (8-10 week old) were anesthetized by intraperitoneal injection of $125 \mathrm{mg} / \mathrm{kg}$ Avertin, then pellets were implanted in the cornea as previously described $[17,18]$. Shh containing pellets were implanted in one eye and control pellets without Shh were implanted in the other eye. Mice were excluded from the study if nonspecific angiogenesis was detected in response to the control pellet. 7 days after pellet implantation, mice were injected with $50 \mu \mathrm{L}$ fluorescienBS1-Lectin I (Vector Laboratories Inc.) via tail vein and sacrificed at least 15 min later, then eyes were harvested and fixed with $1 \%$ paraformaldehyde, and corneas were excised and prepared for fluorescent microscopy. Angiogenesis was quantified as previously described [18].

\section{Echocardiographic assessment of cardiac function}

Trans-thoracic two-dimensional measurements were performed with a high-resolution echocardiographic system equipped with a 30-MHz mechanical transducer (VEVO700, VisualSonics Inc., Toronto, Canada). Mice were anesthetized with a mixture of $1.5 \%$ isoflurane and compressed room air (1 L/min). Mice were anchored to a warming platform in a supine position, limbs were taped to the echocardiograph electrodes, and thoraxes cleaned with a chemical hair remover to minimize ultrasound attenuation. Body temperature was monitored with a rectal thermometer probe and maintained at $37^{\circ} \mathrm{C}+/-1{ }^{\circ} \mathrm{C}$. Aquasonic 100 gel (Parker Laboratories), from which all air bubbles had been expelled, was applied to the thorax to optimize the visibility of the cardiac chambers. Two-dimensional, parasternal long-axis and short-axis views were acquired. Fractional shortening (FS, \%) was calculated as $\mathrm{FS} \%=(\mathrm{LVEDD}-\mathrm{LVESD}) / \mathrm{LVEDD} \times 100 \%$.

\section{Mouse cardiac endothelial cell isolation}

Hearts were collected from mice and washed with ice cold saline to remove blood. Rinsed hearts were then minced to 1 $\mathrm{mm}^{3}$ pieces and placed in $5 \mathrm{~mL}$ of collagenase $(2 \mathrm{mg} / \mathrm{mL})$ for $30 \mathrm{~min}$ at $37^{\circ} \mathrm{C}$. Using a $30 \mathrm{~mL}$ syringe attached to a sterile 14-gauge cannula, the suspension was then fully triturated. The triturated suspension was then passed through a $70 \mu \mathrm{m}$ cell strainer and then centrifuged at $400 \times g$ for 8 min at $4{ }^{\circ} \mathrm{C}$ to pellet the cells. Cells were then incubated with anti-CD31magnetic bead complexes at $4{ }^{\circ} \mathrm{C}$ for $30 \mathrm{~min}$. Magnetic beads (Dyanbeads, Invitrogen, Life Technologies, Grand Island, $\mathrm{NY}$ ) were previously complexed with anti-CD31 antibodies to form antibody-bead complexes. Then, cells were selected with a magnetic separator, and resuspended in rinse buffer. This sequence was repeated six times to ensure a highly selected population of cells. The selected cells were resuspended in $1 \mathrm{~mL}$ RNA-STAT-60 (Tel Test Electronic Labs, Inc., Austin, TX) or RIPA buffer for further use.

\section{Western blot analysis}

Expression of Smo from mouse cardiac CD31+ cells was evaluated by SDS PAGE using rabbit polyclonal anti-Smo (Abcam, Cat\# ab72130) and secondary antibody goat antirabbit IgG-horseradish peroxidase (HRP).

\section{Quantitative real-time PCR (qRT-PCR)}

RNA was isolated from cells with RNA-STAT-60 according to manufacturer's instructions. Total RNA was reverse transcribed with a Taqman cDNA synthesis kit (Applied Biosystems, Foster City, CA) and amplification was performed with a Taqman 7500 (Applied Biosystems). The relative expression of each mRNA was calculated by the comparative threshold cycle $\left(\mathrm{C}_{\mathrm{T}}\right)$ method, normalized to endogenouse $18 \mathrm{~S}$ rRNA expression, and expressed as fold change relative to control. Primer and probe sequences are reported in Table 1.

\section{Immunofluorescence microscopy}

Cardiac tissue sections were prepared as previously described [19]. Endothelial cells were stained with rat antiCD31 antibodies (BD Pharmingen Inc, San Diego, CA, USA) and the presence of Smoothened was assessed with rabbit polyclonal anti-Smo (Abcam, Cat\# ab72130). For immunofluorescence microscopy, primary antibodies were resolved with Alexa-Fluor-conjugated secondary antibodies (Invitrogen Corporation, Carlsbad, CA, USA) and nuclei were counterstained with DAPI (1/5000). Slides were imaged using fluorescent microscopy (Zeiss).

\section{Cell culture}

Human umbilical vein endothelial cells (HUVECs) (ATCC, Manassas, VA) were cultured in endothelial complete growth medium-2 (EGM ${ }^{\mathrm{TM}}-2$ ) (Lonza, Basel, Switzerland). NIH 3 T3 embryonic fibroblasts (ATCC) were maintained in $4.5 \mathrm{~g} / \mathrm{L}$ glucose containing Dulbecco's Modified Eagle's Medium (DMEM) supplemented with $10 \%$ fetal calf serum (FCS) and $100 \mathrm{U} / \mathrm{mL}$ streptomycin/penicillin.

\section{Cell proliferation assay}

Subconfluent HUVECs were seeded on 96-well flat-bottomed plates $\left(1 \times 10^{4} /\right.$ well $)$ containing $100 \mu \mathrm{L}$ EGM-2 
Table 1 Primers and probes used for qRT-PCR analyses

\begin{tabular}{|c|c|c|}
\hline Gene & Primer or probe & Sequence \\
\hline \multirow[t]{3}{*}{$18 S$} & Forward primer & 5'-CGGGTCGGGAGTGGGT-3' \\
\hline & Reverse primer & 5'-GAAACGGCTACCACATCCAAG-3' \\
\hline & Probe & 5'- Cal Fluor-TTTGCGCGCCTGCTGCCTT-BHQ-3' \\
\hline \multirow[t]{3}{*}{$P D G F-B$ (Mouse) } & Forward primer & 5'- ACCTCGCCTGCAAGTGTGA-3' \\
\hline & Reverse primer & 5'- TGCTCCCTGGATGTCCCA -3' \\
\hline & Probe & 5'- Fam- AGTGACCCCTCGGCCTGTGACTAGAAGTC -BHQ-3' \\
\hline \multirow[t]{3}{*}{ Shh (mouse) } & Forward primer & 5'-CAGCGACTTCCTCACCTTCCT-3' \\
\hline & Reverse primer & 5'-AGCGTCTCGATCACGTAGAAGAC-3' \\
\hline & Probe & 5'- Fam-ACCGCGACGAAGGCGCCA-BHQ-3' \\
\hline \multirow[t]{3}{*}{$H G F$ (mouse) } & Forward primer & 5'- CTGACCCAAACATCCGAGTTG-3' \\
\hline & Reverse primer & 5'- TTCCCATTGCCACGATAACAA -3' \\
\hline & Probe & 5'- Fam- TGCTCTCAGATTCCCAAGTGTGACGTGT -BHQ-3' \\
\hline \multirow[t]{3}{*}{$I G F-1$ (mouse) } & Forward primer & 5'- TGCTTCCGGAGCTGTGATCT - $3^{\prime}$ \\
\hline & Reverse primer & 5'- CGGGCTGCTTTTGTAGGCT -3' \\
\hline & Probe & 5'- Fam- AGGAGACTGGAGATGTACTGTGCCCCAC -BHQ-3' \\
\hline \multirow[t]{3}{*}{ Smo (mouse) } & Forward primer & 5'-TTCTTCGTGGGCAGCATTG-3' \\
\hline & Reverse primer & 5'-CCATCTGCTCGGCAAACAA-3' \\
\hline & Probe & 5'- Fam-CTGGCTGGCCCAGTTCATGGATG-BHQ-3' \\
\hline \multirow[t]{3}{*}{$V E G F A$ (mouse) } & Forward primer & 5'-GCAGGCTGCTGTAACGATGA-3' \\
\hline & Reverse primer & 5'-GCATGATCTGCATGGTGATGTT-3' \\
\hline & Probe & 5'- Fam-CCCTGGAGTGCGTGCCCACG-BHQ-3' \\
\hline
\end{tabular}

growth medium. Before the treatment, cells were starved overnight with EBM-2 basal media. Cells were incubated with $100 \mu \mathrm{L}$ DMEM with 0.5\% FCS [20], DMEM with $0.5 \%$ FCS plus Shh $1 \mu \mathrm{g} / \mathrm{mL}$ (Shh), or conditioned media from NIH3T3 fibroblasts treated with $0.5 \%$ FCS and Shh (Shh CM) or without Shh (ctrl CM) for $24 \mathrm{~h}$. Cell proliferation was measured by CellTiter $96^{\circledR}$ AQueous One Solution Cell Proliferation Assay [21] (Promega, Madison, WI) according to the instruction of manufacturer.

\section{Migration assay}

Cell migration was conducted in a modified Boyden's chamber (Neuro Probe, Inc., Bethesda, MD, USA). Briefly, a polycarbonate filter (5- $\mu \mathrm{m}$ pore size) (GE Infrastructure Fairfield, CN, USA) was coated with a solution containing $2.5 \mu \mathrm{g} /$ $\mathrm{mL}$ fibronectin (Sigma-Aldrich Co., St. Louis, MO, USA) and $0.15 \%$ gelatin (Sigma-Aldrich Co.) and inserted between the upper and lower wells, then $5 \times 10^{4}$ cells per well were seeded in the upper chamber. The lower chamber contained DMEM with $0.5 \%$ FCS as negative control, DMEM with $0.5 \%$ FCS plus Shh $1 \mu \mathrm{g} / \mathrm{mL}$ (Curis, Lexington, MA), or conditioned media from NIH3T3 fibroblasts cultured in $0.5 \%$ FCS for $24 \mathrm{~h}$ with (Shh CM) or without (ctrl CM) the addition of Shh $1 \mu \mathrm{g} /$ $\mathrm{mL}$. The chamber was incubated at $5 \% \mathrm{CO} 2$ at $37^{\circ} \mathrm{C}$ for $6 \mathrm{~h}$ and then disassembled and the membrane was stained. The number of cells that had migrated to the lower chamber was counted in 3 high-power fields (HPFs, $\times 20$ magnification) per well. Migration was reported as the mean number of migrated cells per high-power field and normalized to control.

\section{Statistical analysis}

The data are presented as mean \pm s.e. Statistical analysis was performed by using SPSS software (IBM, version 21). Student's $t$ test was performed to compare the means of two groups. One-way ANOVA, followed by the Bonferroni's post hoc test was used to compare the means of multiple groups. Difference was considered statistically significant at $p<0.05$.

\section{Results}

\section{Characterization of endothelial eSmo ${ }^{\text {Null }}$ mice}

To prove effective endothelial-specific Smo knockout, hearts from eSmo ${ }^{\text {Null }}$ and eSmo ${ }^{\mathrm{WT}}$ mice were isolated, collagenase digested, and triturated to form a cell suspension. Endothelial cells were isolated using a CD31 antibody with magnetic bead conjugation and column-based sorting. mRNA and protein expression of Smo from endothelial cells (CD31+ cell fraction) were evaluated by real-time RT-PCR and western blot, respectively. Both mRNA and protein expression of Smo were significant reduced in endothelial cells from 
eSmo $^{\text {Null }}$ mice (Fig.1a, b). Furthermore, the distribution of Smo was assessed by immunofluorescence staining. Smo was present in cardiomyocytes, but was abolished in vascular endothelial cells from eSmo ${ }^{\text {Null }}$ hearts (Fig. 1c).

$\mathrm{eSmo}^{\text {Null }}$ mice exhibited no observable phenotype from birth to age of 1 year. Echocardiography was performed at months $1,4,8$, and 12 to determine whether loss of endothelial Shh signaling resulted in impaired cardiac function, There was no significant difference in fractional shortening (FS) between eSmo ${ }^{\text {Null }}$ and eSmowT mice (Fig. 2).
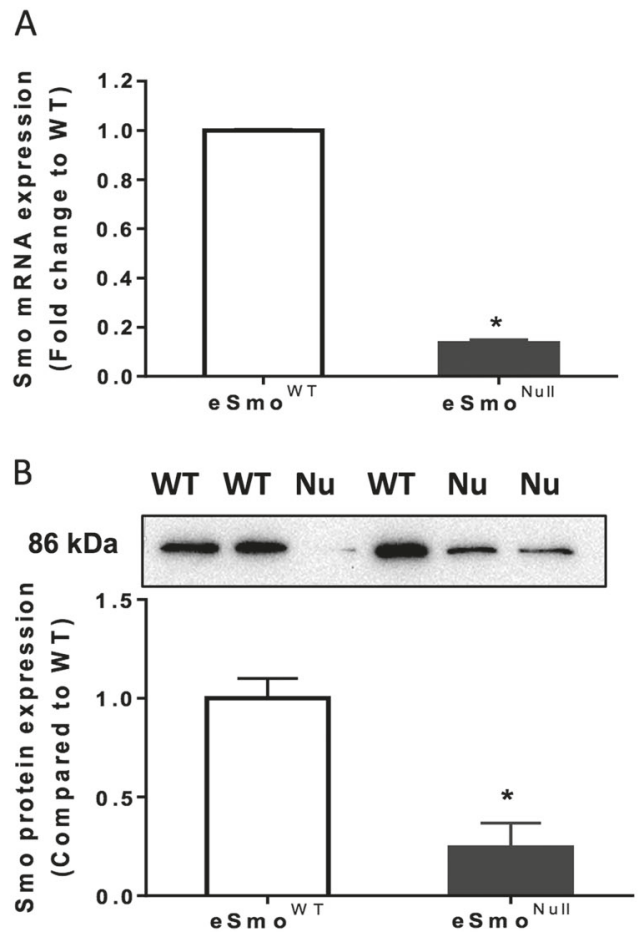

Fig. $1 \mathrm{eSmo}^{\text {Null }}$ mice exhibit decreased Smo expression in primary endothelial cells. eSmo ${ }^{\text {Null }}$ and $\mathrm{eSmo}^{\mathrm{WT}}$ mice $(n=3$ per group) were killed and hearts isolated. Hearts were digested with collagenase and $\mathrm{CD} 31+$ cells were selected with antibody-magnetic bead complexes. a Smo mRNA expression in primary endothelial cells by

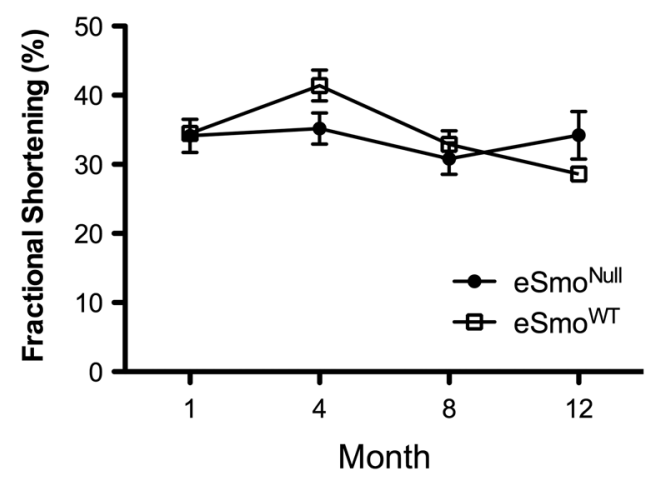

Fig. $2 \mathrm{eSmo}^{\text {Null }}$ mice do not show the alteration in cardiac function up to age 12 months. Murine echocardiography was performed at the indicated time points in eSmo ${ }^{\text {Null }}$ and $\mathrm{eSmo}^{\mathrm{WT}}$ mice. There was no statistically significant difference in fractional shortening $(p>0.05)$

\section{Endothelial Smo-dependent Hedgehog signaling is not required for ischemic tissue repair}

Prior work has demonstrated that Shh is upregulated in the ischemic limb in the HLI model and that blocking Shh with a blocking antibody worsens recovery [7]. Ischemiainduced upregulation of endogenous Shh was confirmed in the HLI model in eSmo ${ }^{\mathrm{WT}}$ mice (Fig.3). Tissue repair after HLI was assessed with Laser Doppler Perfusion Imaging (LDPI), capillary density, Limb motor function and

C
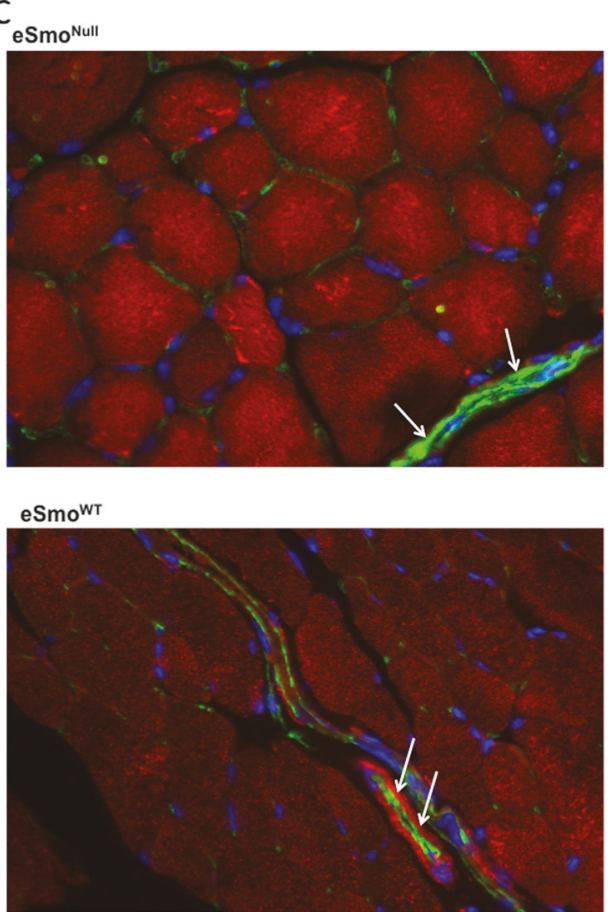

RT-PCR. $(n=3, * p<0.01)$. b Smo protein expression in primary endothelial cells by western blot. $(n=3, * p<0.01)$. c Smo protein distribution in heart sections by immunofluorescence staining. Arrows indicate the endothelial cells. Green: CD31+, Red: Smo, Blue: DAPI (nuclei)

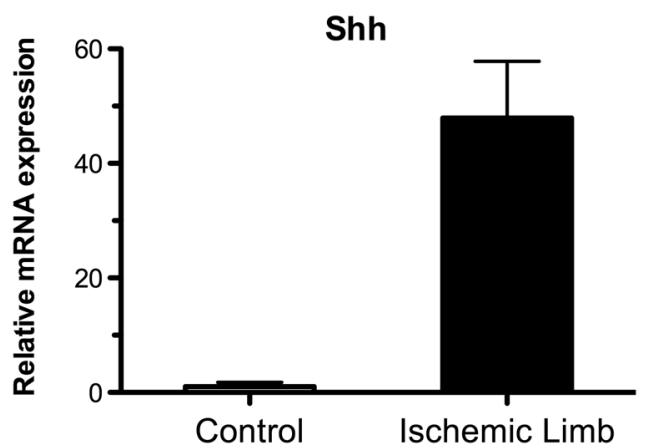

Fig. 3 Shh is significantly induced in the hind limb ischemia model. HLI was performed and mice were sacrificed on day 2 after surgery. Tibialis anterior muscle was excised and RNA was isolated. RT-PCR for Shh was performed. Relative mRNA expression is normalized to the control, nonischemic limb. ${ }^{*} p<0.05$ 
Fig. 4 eSmo $^{\text {Null }}$ mice do not demonstrate deficits in the HLI model. Hind Limb Ischemia (HLI) surgery was performed on $\mathrm{eSmo}^{\text {Null }}$ and eSmo ${ }^{\mathrm{WT}}$ mice ( $n$ $=8-9$ per group). a Laser doppler perfusion imaging (LDPI); (b) Tissue salvage score, and (c) Motor function score were assessed at multiple time points. Mice

were sacrificed for capillary density measurement at 28 days after HLI. d Representative laser doppler perfusion images.

Arrows indicate ischemic limb

Fig. 5 eSmo $^{\text {Null }}$ mice do not show the alteration in capillary density after HLI. Mice were killed for capillary density measurement at 28 days after HLI. a Capillaries were identified as Lectin + cells. Representative images are shown. b Capillary density was assessed as Lectin + cells/HPF and lectin + cells per myocyte fiber
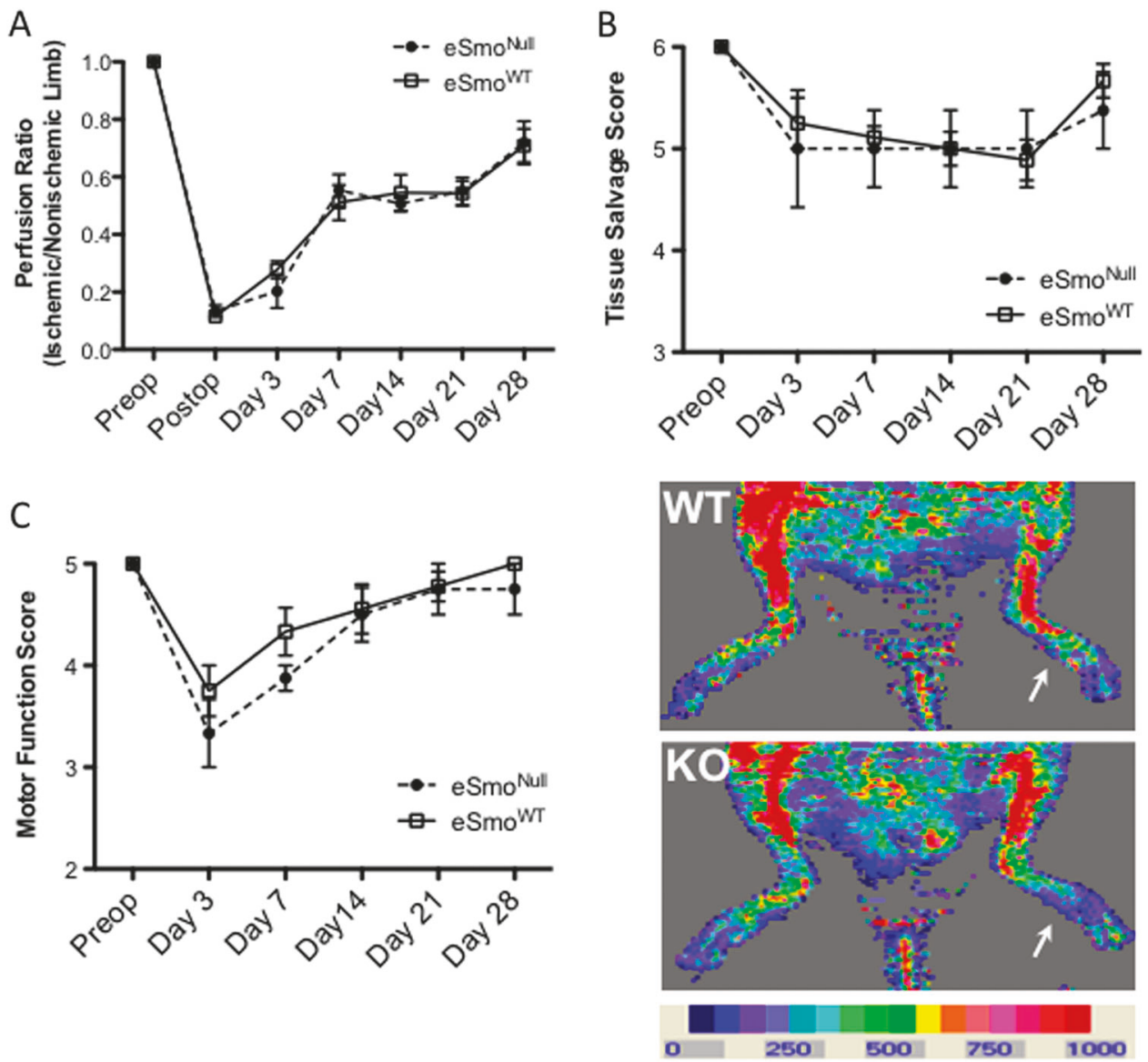

A
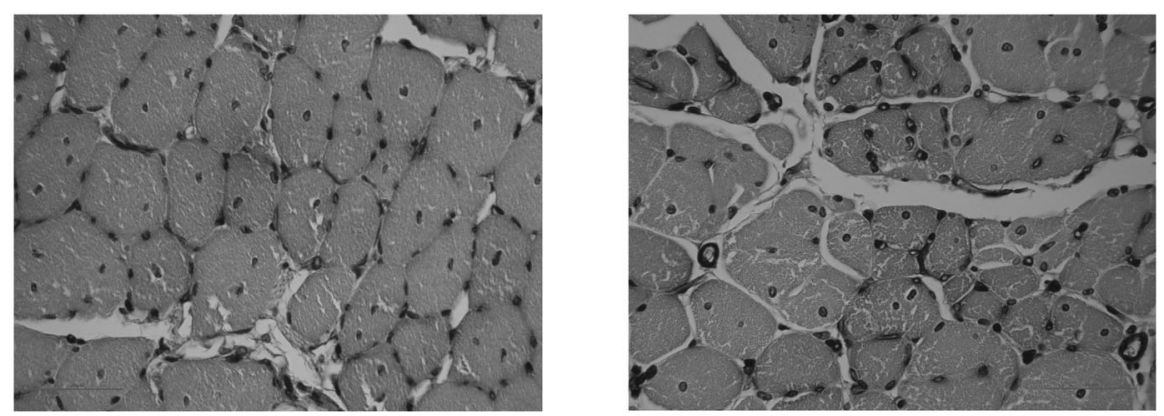

B

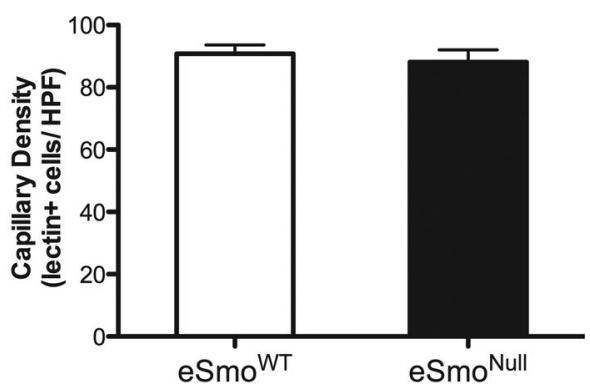

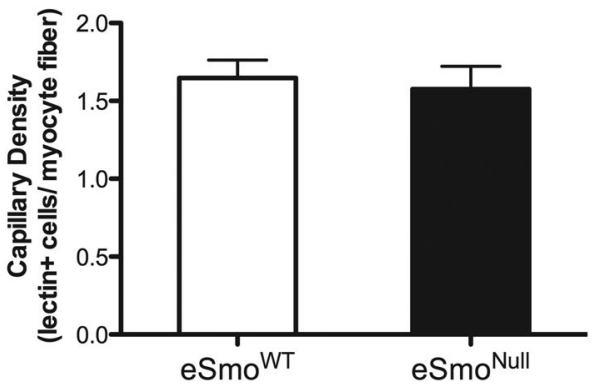

tissue salvage scores (necrosis). Perfusion ratio (ischemic / nonischemic limb), limb motor function, or limb necrosis were not altered in eSmo ${ }^{\text {Null }}$ mice compared with eSmo ${ }^{\text {WT }}$ mice (Fig. 4). Furthermore, there was no significant alteration in capillary density in ischemic limb between $\mathrm{eSmo}^{\text {Null }}$ and $\mathrm{eSmo}^{\mathrm{WT}}$ mice at day 28 after HLI (Fig. 5). 
Fig. 6 eSmo $^{\text {Null }}$ mice do not show the alteration in corneal angiogenesis in response to Shh. a Corneal pellets containing Shh were implanted in the corneas of $\mathrm{eSmo}^{\mathrm{WT}}$ and eSmo ${ }^{\text {Null }}$ mice.

Shh-induced angiogenesis was assessed 7 days later by injecting mice with $50 \mu \mathrm{L}$ fluorescienBS1-Lectin I 15 min prior to killing and then viewing corneas under fluorescence. $\mathbf{b}$

Angiogenesis was quantified as described previously [16]
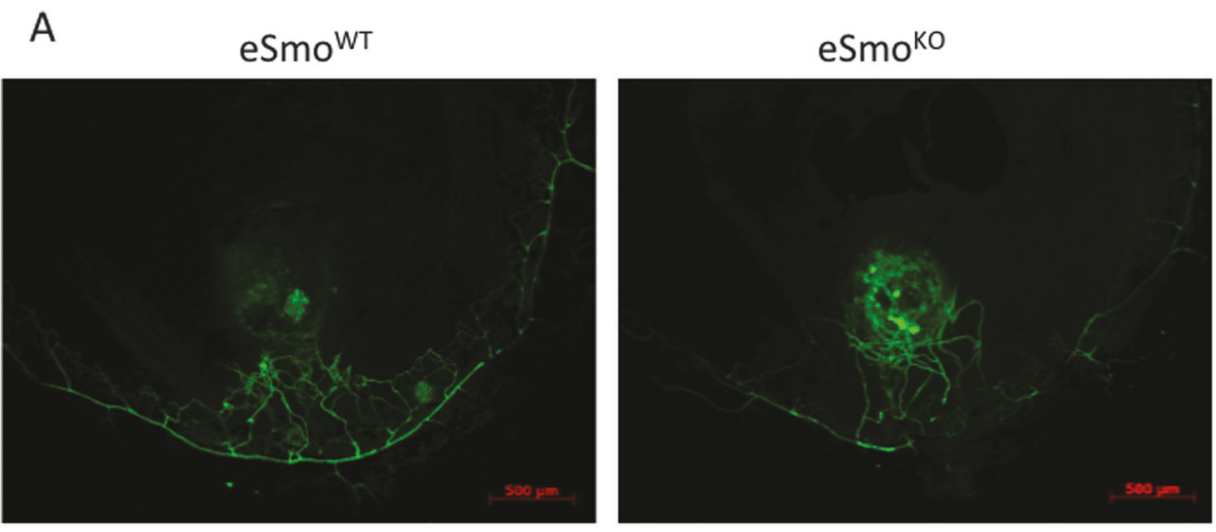

B

Corneal Angiogenesis

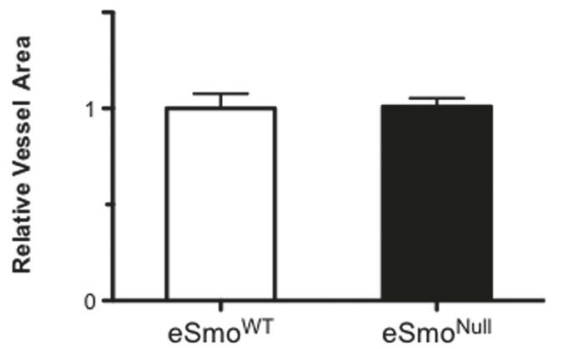

\section{Endothelial Smo-dependent Hedgehog signaling is not required for angiogenesis}

Since the HLI model, which is known to induce endogenous Shh, did not show any difference in recovery between $\mathrm{eSmo}^{\text {Null }}$ and $\mathrm{eSmo}^{\mathrm{WT}}$, we assessed the response to exogenous Shh signaling using the corneal angiogenesis model. Prior work has shown that Shh is an angiogenic factor and induces angiogenesis in the mouse corneal angiogenesis model. Shh or control pellets were implanted into the corneas of $\mathrm{eSmo}^{\text {Null }}$ and $\mathrm{eSmo}^{\mathrm{WT}}$ mice. After 7 days, the degrees of Shh-induced angiogenesis were similar between $\mathrm{eSmo}^{\text {Null }}$ and $\mathrm{eSmo}^{\mathrm{WT}}$ mice (Fig. 6).

\section{Conditional media from Shh-treated fibroblasts promotes endothelial cell proliferation and migration}

Next, we assessed the response of endothelial cell proliferation to Shh. As shown in Fig. 7a, direct Shh had a minimal effect on endothelial cell proliferation. In contrast, conditional media from Shh-stimulated NIH 3T3 fibroblasts had more potent effect on cell proliferation, and this effect was superior to both direct Shh and conditional media from fibroblasts that were not treated with Shh. Next, we assessed endothelial cell migration using a modified Boyden chamber. Once again, conditional media from Shh-treated fibroblasts had most potent effect on endothelial cell migration compared with direct Shh and conditional media from untreated fibroblasts. (Fig. $7 b)$.

To determine the possible mechanism involved in the angiogenic effects observed with conditioned media from Shh-stimulated fibroblasts, we assessed fibroblast gene expression using RT-PCR. Shh significantly stimulated the gene expressions of VEGF-A, PDGF-B, HGF, and IGF-1 in fibroblasts (Fig. 7c-f). PDGF-B was upregulated by about 8 -fold, the highest stimulation amongst the angiogenic growth factors assessed.

\section{Discussion}

Given the established role of Shh in angiogenesis, vasculogenesis, and ischemic tissue repair, the current study investigated the role of endothelial Smo-dependent Hedgehog signaling in a tissue-specific genetically modified mouse model utilizing the Cre-Lox system. Tie2-Cre animals were bred with Smo flox/flox mice to generate endothelial Smo knockout mice. Surprisingly, we did not detect a deficient phenotype either at baseline or in mouse models of corneal angiogenesis and hind limb ischemia. Although it has been clearly established that inhibition of global hedgehog signaling with a blocking antibody results in deficient recovery from ischemic injury [7], the current findings demonstrate that endothelial Smo-dependent Hedgehog signaling is not required for recovery from ischemic injury or Shh-induced angiogenesis. 


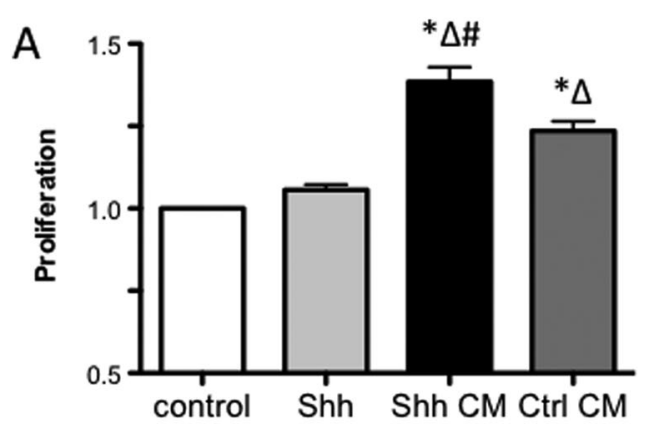

C

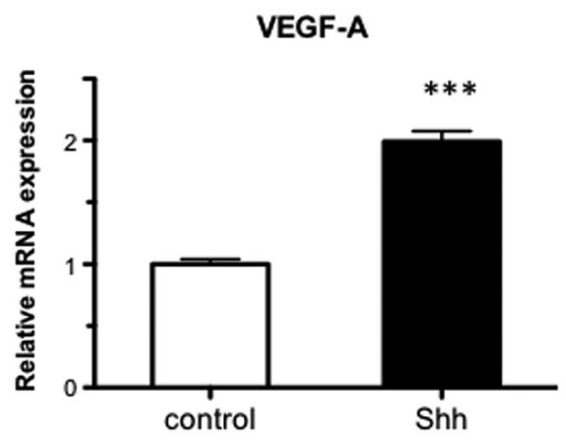

$\mathrm{E}$

HGF

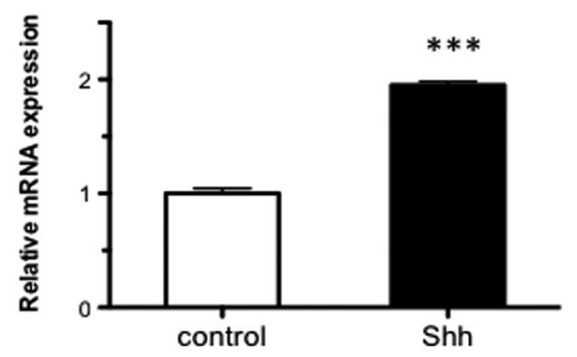

Fig. 7 Conditioned media from Shh-treated NIH3T3 fibroblasts (Shh $\mathrm{CM})$ stimulates greater HUVEC proliferation and migration than direct Shh or control conditioned media (Ctrl CM). Shh conditioned media was created by treating NIH3T3 fibroblasts with Shh $1 \mu \mathrm{g} / \mathrm{mL}$ in DMEM with $0.5 \%$ FCS for $24 \mathrm{~h}$. Control conditioned media was identical except for the absence of added Shh. a Serum-starved HUVECs were treated for $24 \mathrm{~h}$ and then HUVEC proliferation was measured with the MTS assay. b HUVECs were seeded in the upper

Prior findings have demonstrated direct effects of Shh on endothelial cells via non-canonical hedgehog signaling (Ptc$1 /$ Smo-dependent/Gli-independent) in vitro $[9,10]$. Conversely, this study indicates that the greater portion of the Hedgehog signaling effect on angiogenesis in vivo is mediated through activation of non-endothelial cells. Although endothelial cells indeed express the Sonic Hedgehog receptor, Ptc-1, and the Hedgehog signal transducer, Smo, an intact Hedgehog signaling system in endothelial cells does not appear to be required for recovery after ischemic injury or for Shh-mediated angiogenesis.

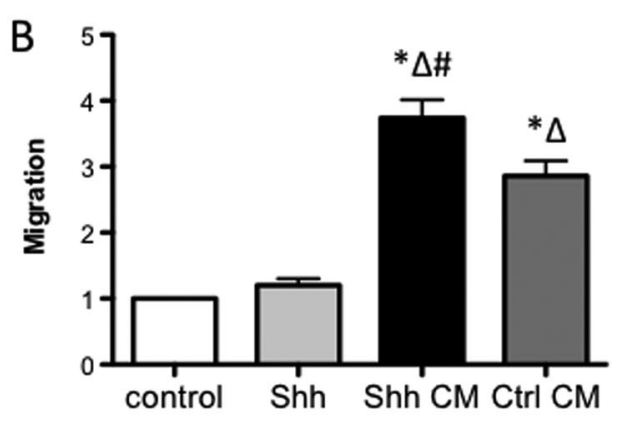

D

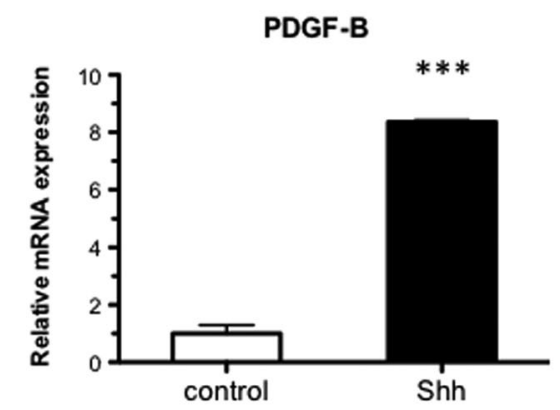

$\mathrm{F}$

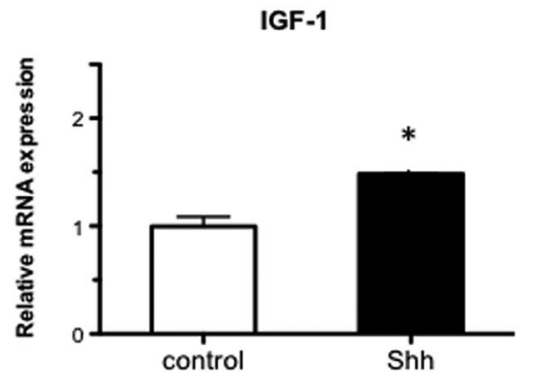

chamber of a modified Boyden chamber and the bottom chamber was filled with the indicated test conditions. The data are normalized to control. $* p<0.01$ vs. control, $\Delta \mathrm{p}<0.01$ vs. Shh, $\# p<0.05$ vs. Ctrl CM. c-f NIH3T3 fibroblasts were treated with Shh $1 \mu \mathrm{g} / \mathrm{mL}$ or control media without Shh for $24 \mathrm{~h}$ and then RNA was collected for RT-PCR. Relative mRNA expression is normalized to control. $* p<0.01$ vs. control, $* * * p<0.001$ vs. control

Importantly, our work addresses a central concern of hedgehog signaling and endothelial biology, namely that Shh demonstrates robust in vivo effects on angiogenesis and ischemic tissue repair, yet direct Shh on endothelial cells in cell culture demonstrates limited effects. In contrast to fibroblasts and other stromal cells, which are known to respond to Shh via the canonical signaling pathway (i.e. Ptc-1/Smo/Gli), prior publications have shown limited effect on Gli signaling when Shh is applied to endothelial cells in vitro. This has led to the postulation of a noncanonical hedgehog signaling pathway operating in 
endothelial cells $[10,22,23]$. Our work demonstrates that, in vivo, endothelial Smo is dispensable for ischemic tissue repair and Shh-induced angiogenesis.

Intriguingly, in the original report by Pola et al. [6]. which demonstrates that Shh is an angiogenic agent, it was noted that Shh treatment resulted in larger and more complex neo-vessels when compared with VEGF. Our finding that PDGF-B is significant upregulated by Shh (Fig. 7d) may explain the previous observations, since PDGF-B regulates pericyte recruitment and pericytes are critically involved in the maturation of neo-vessels [24-26]. Furthermore, prior work has demonstrated that fibroblasts respond to hedgehog ligands in a Smodependent manner and can be inhibited by cyclopamine, a pharmacologic Smo inhibitor [27]. These prior findings also demonstrated that fibroblasts respond to hedgehog ligand via the canonical signaling pathway with activation of Gli transcription factors. Based on these prior findings, it is likely that the gene expression response to Shh demonstrated in NIH3T3 fibroblasts (Fig. 7c-f) occurs via canonical hedgehog signaling. Taken together, these prior findings with our current work indicate that stromal cells have robust responses to hedgehog signaling and elaborate a secondary signaling cascade to achieve angiogenesis and ischemic tissue repair.

There are a number of limitations in this study. First, Tie2-Cre transgenic mice are a genetic tool for the analyses of endothelial cell-lineage gene targeting, however, this promoter is not entirely endothelial specific since others have demonstrated myeloid expression of Tie2 [14]. [28], Nonetheless, we did not find any impairment of angiogenesis or ischemic tissue repair in the Tie2-Cre Smo flox/flox mice. Therefore, the question of myeloid vs. endothelial cell type specificity is less relevant. Regarding the experiments demonstrating the endothelial-specific Smo knockout, there was a small amount of residual Smo expression from isolated primary cardiac endothelial cells using a CD31 antibody (Fig. 1a, b). We believe this was mainly residual contamination from other cell types during the isolation procedure. Furthermore, immunofluorescence staining of cardiac sections clearly demonstrated Smo was only present in vessels of wild type mice but not knockout mice (Fig. 1c).

Overall, this study demonstrates that the angiogenic effect of Shh signaling is independent of an intact endothelial Hedgehog signaling system in vivo. Instead, the results from conditioned media from fibroblasts support the hypothesis that activation of stromal cells and consequent secretion of angiogenic proteins and growth factors by Shh is sufficient to trigger angiogenesis in endothelial cells. Despite the previously established angiogenic effect of Shh, the mechanism of this effect is not via endothelial Smodependent Hedgehog signaling. Along with other reports [29], these findings suggest that Shh mediates angiogenesis through Shh signaling in stromal cells, and focus future attention on the stromal compartment.
Acknowledgements This study was supported in part by grants from the NIH, including F32 HL099283 (R.G.) and HL091983, HL126186, HL053354 and HL134608 (RK) and a grant from the American Heart Association, grant 09POST2230297 (RG).

\section{Compliance with Ethical Standards}

Conflict of interest :Losordo is an employee of Caladrius Biosciences.

\section{References}

1. Varjosalo M, Taipale J. Hedgehog: functions and mechanisms. Genes Dev. 2008;22:2454-72.

2. Chiang C, Litingtung Y, Lee E, et al. Cyclopia and defective axial patterning in mice lacking Sonic hedgehog gene function. Nature. 1996;383:407-13

3. Byrd N, Becker S, Maye P, et al. Hedgehog is required for murine yolk sac angiogenesis. Development. 2002;129:361-72.

4. Washington Smoak I, Byrd NA, Abu-Issa R, et al. Sonic hedgehog is required for cardiac outflow tract and neural crest cell development. Dev Biol. 2005;283:357-72.

5. Agarwal S, Loder S, Cholok D, et al. Local and circulating endothelial cells undergo endothelial to mesenchymal transition (EndMT) in response to musculoskeletal injury. Sci Rep. 2016;6:32514.

6. Pola R, Ling LE, Silver M, et al. The morphogen Sonic hedgehog is an indirect angiogenic agent upregulating two families of angiogenic growth factors. Nat Med. 2001;7:706-11.

7. Pola R, Ling LE, Aprahamian TR, et al. Postnatal recapitulation of embryonic hedgehog pathway in response to skeletal muscle ischemia. Circulation. 2003;108:479-85.

8. Stecca B, Mas C, Clement V, et al. Melanomas require HEDGEHOG-GLI signaling regulated by interactions between GLI1 and the RAS-MEK/AKT pathways. Proc Natl Acad Sci USA. 2007;104:5895-900.

9. Renault MA, Roncalli J, Tongers J, et al. Sonic hedgehog induces angiogenesis via Rho kinase-dependent signaling in endothelial cells. J Mol Cell Cardiol. 2010;49:490-8.

10. Chinchilla P, Xiao L, Kazanietz MG, Riobo NA. Hedgehog proteins activate pro-angiogenic responses in endothelial cells through non-canonical signaling pathways. Cell Cycle. 2010;9:570-79.

11. Rimkus TK, Carpenter RL, Qasem S, Chan M, Lo HW. Targeting the sonic hedgehog signaling pathway: review of smoothened and GLI inhibitors. Cancers. 2016;8:E22.

12. Byrne EF, Sircar R, Miller PS, et al. Structural basis of Smoothened regulation by its extracellular domains. Nature. 2016;535:517-22.

13. Kusano KF, Pola R, Murayama T, et al. Sonic hedgehog myocardial gene therapy: tissue repair through transient reconstitution of embryonic signaling. Nat Med. 2005;11:1197-204.

14. Kisanuki YY, Hammer RE, Miyazaki J, Williams SC, Richardson JA, Yanagisawa M. Tie2-Cre transgenic mice: a new model for endothelial cell-lineage analysis in vivo. Dev Biol. 2001;230:230-42.

15. Long F, Zhang XM, Karp S, Yang Y, McMahon AP. Genetic manipulation of hedgehog signaling in the endochondral skeleton reveals a direct role in the regulation of chondrocyte proliferation. Development. 2001;128:5099-108.

16. Couffinhal T, Silver M, Zheng LP, Kearney M, Witzenbichler B, Isner JM. Mouse model of angiogenesis. Am J Pathol. 1998;152:1667-79.

17. Kenyon BM, Voest EE, Chen CC, Flynn E, Folkman J, D’Amato RJ. A model of angiogenesis in the mouse cornea. Invest Ophthalmol Vis Sci. 1996;37:1652-32. 
18. Rogers MS, Birsner AE, D'Amato RJ. The mouse cornea micropocket angiogenesis assay. Nat Protoc. 2007;2:2545-50.

19. Mackie AR, Klyachko E, Thorne T, et al. Sonic hedgehogmodified human $\mathrm{CD} 34+$ cells preserve cardiac function after acute myocardial infarction. Circ Res. 2012;111:312-21.

20. Group TDCaCTR. The effect of intensive treatment of diabetes on the development and progression of long-term complications in insulin-dependent diabetes mellitus. $\mathrm{N}$ Engl $\mathrm{J}$ Med. 1993;329:977-88.

21. Wary KK, Thakker GD, Humtsoe JO, Yang J. Analysis of VEGFresponsive genes involved in the activation of endothelial cells. Mol Cancer. 2003;2:25.

22. Brennan D, Chen X, Cheng L, Mahoney M, Riobo NA. Noncanonical Hedgehog signaling. Vitam Horm. 2012;88:55-72.

23. Aberger F, Kern D, Greil R, Hartmann TN. Canonical and noncanonical Hedgehog/GLI signaling in hematological malignancies. Vitam Horm. 2012;88:25-54.

24. Hellstrom M, Kal nM, Lindahl P, Abramsson A, Betsholtz C. Role of PDGF-B and PDGFR-beta in recruitment of vascular smooth muscle cells and pericytes during embryonic blood vessel formation in the mouse. Development. 1999;126:3047-55.

25. Rajkumar VS, Shiwen X, Bostrom M, et al. Platelet-derived growth factor-beta receptor activation is essential for fibroblast and pericyte recruitment during cutaneous wound healing. Am J Pathol. 2006;169:2254-65.

26. Lindahl P, Johansson BR, Leveen P, Betsholtz C. Pericyte loss and microaneurysm formation in PDGF-B-deficient mice. Science. 1997;277:242-5.

27. Taipale J, Chen JK, Cooper MK, et al. Effects of oncogenic mutations in Smoothened and Patched can be reversed by cyclopamine. Nature. 2000;406:1005-9.

28. Bailey AS, Willenbring H, Jiang S, et al. Myeloid lineage progenitors give rise to vascular endothelium. Proc Natl Acad Sci USA. 2006;103:13156-61.

29. Chen W, Tang T, Eastham-Anderson J, et al. Canonical hedgehog signaling augments tumor angiogenesis by induction of VEGF-A in stromal perivascular cells. Proc Natl Acad Sci USA. 2011;108:9589-94. 